\title{
Intuition und Erkenntnis
}

Im folgenden Schlusskapitel sollen die verschiedenen Stränge der Untersuchungen zu Bestimmung und epistemischem Status der Intuition miteinander verknüpft werden. Diese Verknüpfung wird maßgeblich vom Konzept der mentalen Modelle ausgehen, da sich auf dessen Grundlage sowohl die klassischen als auch die modernen Intuitionsverständnisse und sowohl die epistemologischen als auch die psychologischen Befunde einordnen lassen. Zunächst soll der Mentale-Modelle-Ansatz zu den klassisch-philosophischen Intuitionsverständnissen, welche der Ausgangspunkt der Untersuchung waren, in Bezug gesetzt werden. Auf dieser Grundlage kann anschließend Bilanz darüber gezogen werden, was in begriffsbestimmender und epistemologischer Hinsicht erreicht wurde.

\subsection{Rückbezug auf philosophische Positionen}

Aus der Diskussion der klassischen Positionen zur Intuition sollen im Folgenden drei Kernthemen herausgegriffen und aufgrund des nun Erarbeiteten nochmals betrachtet werden: Gewissheit vs. Sicherheit, die Frage der Erkenntnis des Wesens sowie die Kantische Perspektive auf Intuition und Imagination. Die Leitfrage dabei ist, inwiefern das auf psychologischen Grundlagen erarbeitete Intuitionsverständnis mit seinen epistemischen Implikationen die klassisch-philosophischen Positionen bestätigt, ergänzt oder korrigiert.

\subsubsection{Descartes: Gewissheit vs. Sicherheit}

Ein Thema, welches bereits bei Descartes und auch im Verlauf dieser Arbeit zentral war, ist der epistemische Status der Intuitionen. Hier wurden die phänomenalen Merkmale der Unmittelbarkeit und Gewissheit aus den Positionen und Beispielen zur Intuition herausgearbeitet. Doch entspricht der Gewissheit auch eine Sicherheit? Zwar bezeichnete Descartes noch in den Regulae die intuitive Erkenntnis als diejenige, bei der über das Erkannte kein Zweifel zurückbleibt. Hierbei erfolgte der Verweis auf die Einsicht in selbstevidente Wahrheiten. Dennoch konnte festgestellt werden, dass es für Descartes ein Problem darstellt, ob der Intuition auch eine Tatsache entspricht.

Eine erste, einfache Reaktion auf diese Frage besteht darin, auf die Fallibilität der Intuition hinzuweisen, die für alle menschliche Erkenntnis schlicht 
nicht mehr von der Hand zu weisen ist. Doch soll das Verhältnis des hier vorgelegten Intuitionsverständnisses zu Descartes' Ausführungen noch etwas genauer betrachtet werden. Hilfreich hierfür ist es sich zu vergegenwärtigen, welche zwei Arten von Intuition bei Descartes vorkommen: Erstens kennt Descartes die Intuition als Einsicht in selbstevidente Wahrheiten. Bei dem Versuch, diese Wahrheiten weiter zu begründen, kommt man nicht weiter; denn erkennt man die entsprechende Wahrheit, ist sie bereits aus sich selbst heraus begründet. Zweitens beschreibt Descartes die Intuition im Zusammenhang mit den Schritten einer Deduktion: Jeder der kleinsten Schritte in einer deduktiven Kette muss intuitiv erkennbar sein; es gibt also intuitive Schlüsse.

Gemäß dem vorliegenden Verständnis beruht Intuition auf Typ 1Denkprozessen, die von zumeist in mentalen Modellen integrierten Gehalten ausgehen und zu anderen Gehalten (= Intuitionen ${ }_{N P}$ ) hinführen. Dies ist ohne Schwierigkeiten auf selbstevidente Wahrheiten im Sinne der Cartesischen Anfangsgründe sowie auf die intuitiven Übergänge in deduktiven Ketten anwendbar. Genauer können wir die beiden Cartesischen Intuitionsarten wie folgt auffassen: Die Einsicht in selbstevidente Wahrheiten (= Anfangsgründe) entspricht dem Fall, in dem man auf einen (in der Regel in einem mentalen Modell eingebetteten) Gehalt stößt. >Darauf stoßen< ist ein passendes Sprachbild, da der Gehalt oder die im mentalen Modell angeordnete Gehalte eventuell auch durch ihre Anordnung - sich selber oder einander begründen. Es besteht somit weder die Notwendigkeit noch die Möglichkeit einer weiteren Begründung dieses Gehalts. Betrachten wir dies anhand des mentalen Modells $<$ ich denke>: Bestandteil dieses Modells ist, dass man denkt, sobald man es denkt. Sofern Descartes Recht hat, ist also im Denken selber eine Metaperspektive auf das Denken enthalten. Nun ergibt sich die Möglichkeit, dass ein weiteres mentales Modell hinzugezogen wird, welches eine neue Verbindung herstellt - hier zwischen der erwähnten in <ich denke> enthaltenen Metaperspektive und der Tatsache der eigenen Existenz. ${ }^{392}$ Jedoch wurde bereits zuvor, bei der Selbstevidenz von <ich denke>, von >Ableitung < gesprochen, da auch hier etwas implizit im mentalen Modell enthalten ist, was zunächst abgelesen werden muss. Dies führt uns zur Einsicht, dass zwischen den beiden Intuitionsarten bei Descartes - der intuitiven Erkenntnis der Anfangsgründe und den intuitiven Schlüssen - kein bedeutsamer Unterschied besteht. Ob ein weiteres mentales Modell hinzuzuziehen ist oder nicht, wird im Einzelfall

392 Hier ist genau genommen nicht von Descartes' in den Mediationes artikulierter cogito-Intuition die Rede, sondern von einer alltäglichen, d.h. unreflektierten intuitiven Ableitung der Existenz aus dem Denken. Demgegenüber bezieht sich Descartes' Intuition bereits auf das Folgen des Denkens aus der Existenz (siehe Kap. 4.5.2). 
entschieden und ist möglicherweise bis zu einem gewissen Grad willkürlich (wo ziehen wir die Grenze zwischen verschiedenen mentalen Modellen, was betrachten wir noch als ein einziges Modell?).

Eine weitere Konsequenz des hier Vorgebrachten ist vermutlich die weitgehende Irrelevanz der a priori-a posteriori-Unterscheidung in Bezug auf die Funktionsweisen der Intuition. Dies wird etwa dann ersichtlich, wenn >ich denke, ich existiere « mit der Intuition der Feuerwehrkommandantin verglichen wird. Auf der funktionalen Ebene ergibt sich hier kein relevanter Unterschied; in beiden Fällen liest das Subjekt (auf der Ebene des Typ 1-Denkens) ab, was bereits in seinem mentalen Modell bzw. seinen mentalen Modellen enthalten ist. Das mentale Modell <ich denke> ist ebenso eine ikonische Repräsentation eines Subjekts wie das mentale Modell des Brandplatzes. Allenfalls kann der Unterschied auf diskursiver Ebene, also wenn wir begrifflich über den $\mathrm{Zu}$ sammenhang zwischen Denken und Existenz nachdenken, eine Rolle spielen (z.B., wenn wir die Auffassung vertreten, dass der Begriff des Denkens notwendigerweise denjenigen der Existenz in sich enthält). In diesem Fall geben wir der a priori-a posteriori-Unterscheidung eine auf das Verhältnis von Begriffen und Tatsachen bezogene Bedeutung, an welcher sich bestimmt festhalten lässt. Beschreiben wir jedoch die Intuition als mentalen Zustand, bringt uns die a priori-aposteriori-Unterscheidung keinen Erkenntnisgewinn für diese Beschreibung, da diese Unterscheidung auf der Ebene des Gehalts, nicht des mentalen Zustands anzusiedeln ist. Genauso wie sich eine Überzeugung auf a priori- oder a posteriori-Gehalte beziehen kann, gilt dies auch für die Intuition.

Was bedeutet nun all dies in Bezug auf die Frage Gewissheit vs. Sicherheit der Intuition? Kehren wir zurück zum Begriff der Selbstevidenz: Die obigen Ausführungen sollten deutlich gemacht haben, dass zunächst keine Intuition sselbstevidenter ist als die andere: Immer geht es darum, dass aus einem bestehenden mentalen Modell bestimmte Gehalte abgelesen werden, was zu Intuitionen führt. Intuitionen bleiben aber in jedem Fall realitätssensitiv, wie in der Abgrenzung zur Imagination festgestellt wurde. Dies erklärt das phänomenale Merkmal der Gewissheit, welches mit der Intuition verbunden auftritt. Vorhandene mentale Modelle werden im Vorgang der Intuition abgelesen und eröffnen so den Weg zu neuer Erkenntnis aus dem, was bereits auf einer tieferen Erkenntnisebene registriert wurde. Ob diese Registrierung »Abbild « der Realität ist oder nicht, wird über die Korrektheit der Intuitionen entscheiden. Dies kann und muss zu einer Differenzierung des Erkenntnisbegriffes führen: Erkannt wird mit der Intuition in jedem Fall; ob die Wirklichkeit erkannt wird und somit in Cartesischer Terminologie der Gewissheit eine Sicherheit entspricht, entscheidet sich an anderer Stelle. Auch eine eingehendere Betrachtung bestätigt also, was bereits provisorisch festgehalten 
wurde: Die Intuition ist, obwohl sie mit erlebter Gewissheit einhergeht, keine privilegierte Erkenntnisgattung, da auch sie darauf angewiesen ist, dass die mentalen Modelle, auf denen sie basiert, korrekte Repräsentationen der Wirklichkeit sind. Bei selbstevidenten Wahrheiten ist diese Korrektheit eben selbstevident, dies hat aber keine spezifische Verbindung zur Intuition als Erkenntnisgattung.

Descartes' Ausführungen zur Intuition sind trotz dieser Einschränkungen höchst wertvoll, da sie insbesondere auf der phänomenalen Ebene viel zur Klärung des Intuitionsverständnisses beigetragen haben. So konnten insbesondere dank Descartes' Beschreibungen und Reflexionen Unmittelbarkeit und Gewissheit als phänomenale Merkmale der Intuition bestimmt werden (Kap. 3.1). Außerdem dürfen seine Beispiele zur Anwendung von Intuitionen allen voran >ich denke, ich existiere < - nach wie vor als paradigmatisch für viele philosophische Intuitionsverständnisse gelten. Sie sind daher ein Prüfstein geblieben, an dem sich auch das hier entwickelte Intuitionsverständnis bewähren muss.

\subsubsection{Spinoza und Goethe: Erkenntnis des Wesens und Wesensbegriffe}

Zunächst eine kurze Repetition der dargestellten Positionen zum Thema der Wesenserkenntnis: Laut Spinoza kann mittels Intuition Wesenserkenntnis erfolgen. Mit der Unterscheidung der Erkenntnisarten (opinio/imaginatio, ratio und scientia intuitiva) weist er darauf hin, dass zwar sowohl auf deduktivem als auch auf intuitivem Weg adäquate Erkenntnis erlangt werden kann, die Resultate sich aber voneinander unterscheiden: Während die ratio Gemeinbegriffe hervorbringt (notiones communes), kommen wir mittels scientia intuitiva zu Wesensbegriffen (notiones essentiae rerum), also zur Einsicht in das Wesen der Einzeldinge. Kant knüpft an Spinozas Unterscheidung an, verneint aber, dass uns letztere Erkenntnis möglich ist, da wir nicht über einen anschauenden Verstand (intellectus archetypus) verfügen. Dementsprechend sind gemäß Kant alle Begriffe Gemeinbegriffe, wie auch alle menschliche Erkenntnis diskursiv ist. Goethe nimmt die Thematik wieder auf und kommt, mit Spinoza und gegen Kant, zur Überzeugung, dass Wesenserkenntnis menschenmöglich ist. Im Unterschied zu Spinoza verortet Goethe diese Erkenntnis jedoch nicht allein im Apriorischen, sondern beginnt mit der diskursiven Methode (vermittelt durch die induktive Aufnahme einzelner empirischer Erscheinungen, wie etwa in seinen Pflanzenstudien). Durch das Studium der einzelnen Erscheinungen und der Übergänge zwischen diesen erschließt sich ihm ein zugrundeliegendes Wesen (z.B. das sideelle Blat‘, die Urpflanze). Dieser Wesensbegriff unterscheidet sich vom Gemeinbegriff dadurch, dass die Begriffssetzung nicht willkürlich, aufgrund einer vorgängigen Bestimmung gemeinsamer Merkmale, erfolgt (wie in Kants Beispiel des Begriffs $>$ Baum $<$ ), 
sondern unwillkürlich ins Bewusstsein tritt. Hierzu passen die phänomenalen Merkmale der Intuition: Unmittelbarkeit und Gewissheit.

Die Goethesche Einsicht in einen Wesensbegriff, wie z.B. in denjenigen der Urpflanze, gleicht den später in der Arbeit vorgebrachten Beispielen intuitiver Erkenntnis: z.B. der Erkenntnis der Ermittlerin, die von der Wand mit den Tatortfotos und -skizzen einen Schritt zurücktritt, Kekulés Entdeckung des Benzolrings oder der Expertenintuition der Feuerwehrkommandantin. Mit den erarbeiteten Grundlagen des Mentale-Modelle-Ansatzes, namentlich der Zeichentheorie Peirces, können wir Goethes Methode nun in einen wissenschaftstheoretischen Rahmen stellen. Dies soll über den Weg eines kurzen Exkurses zum Zusammenhang zwischen Abduktion und Mentalen Modellen und der Intuition unternommen werden.

Peirce fügt an seine Ausführungen zu ikonischen Zeichen und Diagrammen eine einflussreich gewordene Unterscheidung zwischen Arten des Schließens an, die sich insbesondere auf wissenschaftliche Methoden anwenden lassen: Peirce unterscheidet zwischen abduktiven, deduktiven und induktiven Schlüssen. ${ }^{393}$ Während der deduktive Schluss nicht-ampliativ (d.h. nicht erkenntniserweiternd) ist, sind der induktive sowie der abduktive Schluss ampliativ, womit zugleich gegeben ist, dass deren Konklusion, bzw. das Resultat desSchlusses, nicht zwingend aus den Prämissen folgt. Sowohl bei der Induktion als auch bei der Abduktion wird von einem Einzelfall auf das Allgemeine geschlossen. ${ }^{394}$ Während aber bei der Induktion gute Gründe für diesen Schluss explizit vorliegen (z.B. häufiges Auftreten) ist dies bei der Abduktion nicht der Fall. Zugleich ist die Abduktion im Unterschied zu Induktion und Deduktion ein kreatives Verfahren: Peirce zufolge ist die Abduktion die einzige logische Operation, die neue Ideen einführen kann. ${ }^{395}$ Dadurch kommt der Abduktion im wissenschaftlichen Prozess die Aufgabe der Hypothesenbildung zu. ${ }^{396}$

393 Vgl. Peirce (CP, § 2.623).

394 Auch aufgrund dieser Gemeinsamkeit ist die Abgrenzung zwischen Abduktion und Induktion bisweilen problematisch und ist in Peirces frühen Schriften nicht völlig klar (vgl. Park 2016, S. 19-22).

395 Vgl. CP, § 2.623, sowie EP, S. 287.

396 Bekannt geworden ist in diesem Zusammenhang Peirces Schilderung der Abfolge der drei Schlussverfahren im wissenschaftlichen Prozess: Durch Abduktion wird eine Hypothese aufgestellt, danach müssen aber Deduktion und Induktion einsetzen:

Deduction produces from the conclusion of Abduction predictions as to what would be found true in experience in case that conclusion were realized. Now comes the work of Induction, which is not to be done while lolling in an easy chair, since it consists in actually going to work and making the experiments, thence going on to settle a general conclusion as to how far the hypothesis holds good.

(EP, S. 288) 
Abduktion steht mit Peirces Überlegungen zu ikonischer Repräsentation und Diagrammen in engem Zusammenhang. Insofern die Abduktion bei Peirce ein kreatives, horizonterweiterndes Verfahren ist, kann sie in besonderer Weise auf ikonische Repräsentation zurückgreifen. Als Schlussverfahren, so Peirce, ist die Abduktion grundsätzlich symbolischer Natur. Doch wie wir im Fall der Diagramme gesehen haben, können Repräsentationen symbolische und ikonische Aspekte zugleich enthalten. Peirce beschreibt den ikonischen Aspekt der Abduktion wie folgt:

An originary Argument, or Abduction, is an argument which presents facts in its Premiss which present a similarity to the fact stated in the Conclusion, but which could perfectly well be true without the latter being so, much more without its being recognized; so that we are not led to assert the Conclusion positively but are only inclined toward admitting it as representing a fact of which the facts of the Premiss constitute an Icon. .97

Der ikonische Aspekt der Abduktion besteht also darin, dass das Resultat dieses Schlusses in einer Ähnlichkeitsrelation zu seinen Voraussetzungen steht. Dies ist zugleich ein Lösungsvorschlag zur Frage der Kreativität: wie Neuentdeckungen und Horizonterweiterungen möglich sind, die doch in $\mathrm{Zu}-$ sammenhang mit vorherigem Wissen stehen. ${ }^{398}$ Wie Peirce betont, findet die Abduktion situiert statt. Dies zeigt sich insbesondere im Bereich der Wissenschaft: Die Hypothese wird nicht im luftleeren Raum generiert, sondern entspringt - wenn auch auf nicht definierte Weise - vorgängigen Erfahrungen. Dies kann etwa bedeuten, dass sich das Subjekt mit einem Problem (meist über längere Zeit und intensiv) beschäftigt hat. In jedem Fall ist die Situierung dadurchgegeben, dass dasSubjektdenFokusauf eineProblemstellungrichtet. ${ }^{399}$

Über die Situierung sind wir wieder bei den mentalen Modellen angelangt. Abduktion lässt sich treffend als model-based reasoning bezeichnen. ${ }^{400}$ Hierbei kann die Abduktion als intuitives Verfahren gelten, d.h. als Verfahren, in dem die Intuition den Part der Horizonterschließung übernimmt. ${ }^{401}$ Umgekehrt

397 CP, § 2.96, sowie auch § 2.623; EP, S. 287 .

398 Vgl. Rohr (1993, S. 91ff.).

399 Vgl. Hintikka (1998, S. 518f.).

400 Vgl. Caterina/Gangle (2016, S. 28).

401 Die hier vorgenommene Zusammenführung von Abduktion und Intuition ist möglicherweise der Kritik ausgesetzt, Peirces Einordnung der Abduktion zuwiderzulaufen. So schließt Anderson aus folgenden Gründen darauf, dass Peirce Abduktion nicht mit Intuition identifiziert: i) Peirce sagt an keiner Stelle, Abduktion sei intuitives Schließen, ii) Intuition gilt traditionellerweise als kontextfrei erlangte Einsicht (mit Verweis auf Descartes), während bei Peirce Abduktion immer kontextgebunden ist, iii) Intuition 
zeigt der Anschluss an die Abduktion, inwiefern Intuition im wissenschaftstheoretischen Kontext bedeutsam werden kann. Vor diesem Hintergrund können Beispiele wie das bereits angeführte Kekulés treffend als Beispiele sowohl für Intuition als auch für Abduktion in den Wissenschaften angeführt werden. ${ }^{402}$

Auch das zuvor etwas mystisch erschienene Beispiel von Goethes naturwissenschaftlichen Studien lässt sich nun überzeugend als wissenschaftliches (oder zumindest wissenschaftsnahes) Vorgehen beschreiben, in dem Abduktion eine zentrale Rolle spielt. In den Pflanzenstudien kann Goethe aus den einzelnen Pflanzen nicht einfach auf induktivem Weg eine allgemeine Regel ableiten, die den Pflanzenstadien zugrunde liegt, da in den einzelnen Beobachtungen die Regel nicht direkt ersichtlich wird. Vielmehr ist der kreative Prozess der Abduktion hier am Werk: Durch die intensive Beschäftigung mit der Thematik, zu der die zahlreichen Einzelbeobachtungen wie auch Goethes Interesse ein zugrundeliegendes Wesen der Pflanze zu finden gehören, bildet Goethe die Hypothese der Urpflanze. Diese als übergeordnete Hypothese zu bezeichnen ist insofern treffend, als sich aus diesem abduktiv erlangten Wesensbegriff nun auf deduktivem Weg einzelne Hypothesen über Pflanzen gewinnen lassen (z.B., eine bestimmte Pflanze wird sich bei ihrem weiteren Wachstum so-und-so weiterentwickeln). Diese Hypothesen wiederum lassen sich auf induktivem Weg überprüfen: Goethe kann und sollte zu den einzelnen Beobachtungen zurückkehren und beurteilen, wie gut sie die aus der Urpflanze abgeleiteten Hypothesen bestätigen. Während die deduktiven und induktiven Schritte zum Begründungszusammenhang der Wissenschaft gehören, ist der am Anfang stehende abduktive Schritt dem Entdeckungszusammenhang zuzuordnen.

ist infallibel, Abduktion gemäß Peirce fallibel (vgl. Anderson 1987, S. 37-39; siehe auch Paavola 2005, S. 143).

Anderson hätte in allen Punkten Recht, wenn dieses traditionell-rationalistische Intuitionsverständnis vorausgesetzt würde. Nach dem hier vorgelegten Ansatz gilt aber: Intuition ist keineswegs kontextfrei und durchaus fallibel. Diese Modifikationen des ursprünglichen Intuitionsbegriffs führen dazu, dass Peirces eigene Ausführungen zum Zusammenhang zwischen Intuition und Abduktion nicht übernommen werden müssen.

402 Da Kekulé via visuelle imagery zu einer Hypothese gelangt, könnte dies vermutlich als ein Beispiel visueller Abduktion eingeordnet werden. Im Unterschied zur herkömmlichen visuellen Abduktion, wie sie etwa bei Magnani (2001, S. 106-115) erläutert wird, wird das Lösungsbild des Benzolrings nicht durch gewolltes Sich-Vorstellen hervorgerufen, sondern es steht Kekulé unmittelbar vor Augen. Wenn Intuitionen zunächst durch Typ 1->Ablesungen< von mentalen Modellen zustande kommen, kann das Konzept der visuellen Abduktion möglicherweise auf diesen Bereich ausgeweitet werden. 
Der Mentale-Modelle-Ansatz und mit ihm die Untersuchung der Intuition als Erkenntnisart sowie auch die Analyse der Abduktion gehören in wissenschaftstheoretischer Hinsicht also der Betrachtung des Entdeckungszusammenhangs an. Auf diesen fokussierend können wir in Goethes Suche nach der Urpflanze die auf mentalen Modellen basierende Intuition am Werk sehen und das Beispiel Goethes mit den Beispielen Kekulés und der Feuerwehrkommandantin parallelisieren. Goethe bildet durch das intensive Pflanzenstudium eine Expertise heraus, die sich als Aufbau eines passenden mentalen Modells beschreiben lässt. Die ihm plötzlich vor dem geistigen Auge stehende Urpflanze erscheint nicht aus dem Nichts, sondern ist in seinem mentalen Modell bereits implizit enthalten. Wie das Diagramm, welches nochmals betrachtet werden kann und dadurch neue Erkenntnis ermöglicht, ist auch dieses mentale Modell der Re-Examination zugänglich. Der Vergleich mit dem Diagramm lässt die einzelnen Beobachtungen als Punkte zwischen bestimmten Koordinaten verstehen, deren Verbindung bei der nochmaligen Betrachtung gezogen werden kann. Dadurch entsteht das, was in Spinozistischer Perspektive als Wesensbegriff galt: die Urpflanze als Hypothese, die auf abduktivem Weg erlangt worden ist.

Der Mentale-Modelle-Ansatz und Peirces Auffassung der Abduktion lassen sich auch in ihrer gemeinsamen Modifikation des ursprünglichen Konzepts der Wesensbegriffe parallelisieren. Wie Peirce betont, ist Abduktion keinesfalls infallibel und entspricht somit gerade nicht der Intuition, wie sie in rationalistischer Perspektive beschrieben wurde. ${ }^{403}$ Vielmehr ist Abduktion ein educated guess; man könnte im Falle Goethes hinzufügen: very educated. Dies reicht aus, um die Untersuchung des Entdeckungszusammenhangs aufwerten. ${ }^{404}$ Wir haben nun die Instrumente zur Hand, um zu verstehen, was in diesen entdeckenden Schritten vor sich geht. Über die Beschreibung des Erkenntnisprozesses hinaus können wir eine externalistische Rechtfertigungsperspektive auf die Intuition einnehmen und beurteilen, als wie zuverlässig diese aufgrund des Subjekts, dessen Umwelt und dessen Handlungen (siehe bei Goethe: dessen intensives Studium der Pflanzen) gelten kann. Wir untersuchen damit insbesondere die Möglichkeiten des Subjekts, korrekte, die Umwelt ikonisch repräsentierende mentale Modelle aufzubauen. Trotz der Abweichungen dieses neueren Ansatzes von Descartes' infallibler Intuition

403 Vgl. Pape (2004, S. 79).

404 Hier lässt sich ein erkenntnistheoretischer Beitrag zum wiedererwachten Interesse am Entdeckungszusammenhang in der Wissenschaftstheorie leisten, was auch zu neueren Untersuchungen über die Abduktion geführt hat (siehe z.B. Hintikka 1998, Schurz 2008, Magnani 2009; vgl. Park 2016, S. 1). 
und Spinozas Verständnis der Wesensbegriffe bleibt der zentrale Unterschied, der sich im Gegensatz zwischen Wesensbegriffen und Gemeinbegriffen gezeigt hat, bestehen: es ist der Unterschied zwischen einem abduktiven, aufgrund der Intuition aus mentalen Modellen Neues erschließenden Erkenntnisvorgang und einem induktiven oder deduktiven, der explizit vorhandene Merkmale zu Begriffen bündelt, bzw. aus Begriffen andere Begriffe ableitet.

Dadurch, dass die Wesensbegriffe vom Merkmal der Infallibilität befreit werden können, ohne deren mit intuitiver Erkenntnis in Verbindung stehende Charakteristik aufgeben zu müssen, lässt sich eine neue Perspektive auf die Problematik offensichtlich falscher oder verzerrter Bildungen von Wesensbegriffen gewinnen. Menschen nutzen ihre mentalen Modelle exzessiv, um aus ihnen neue Informationen zu gewinnen; insbesondere über die ihren Beobachtungen zugrundeliegenden Essenzen. ${ }^{405}$ Dies tut Goethe mit den Pflanzen, dies tun Menschen mit der Gruppierung und über das Beobachtbare hinausgehenden Wesensbeschreibung anderer Menschen, etwa im Fall rassistischer Essentialisierung. Eingedenk solcher Beispiele der Essentialisierung darf man keineswegs annehmen, die intuitiv formierten Wesensbegriffe bildeten immer die Realität ab. Vielmehr drängt sich ein heuristisches Verständnis der Wesensbegriffe auf: Sie sind allgemein nützlich, aber nicht vollständig und nicht immer der Realität entsprechend, bisweilen sogar gefährlich. ${ }^{406}$ Mit dem Mentale-Modelle-Ansatz lässt sich die innere Untersuchung des intuitiven Erkenntnisprozesses von der notwendigerweise beizuziehenden externalistischen Rechtfertigungsperspektive trennen, ohne dabei den intrinsischen Wert der Intuition negieren zu müssen, der sich aus dem Erschließen neuer Erkenntnisse aus vorhandenen mentalen Modellen ergibt.

\subsubsection{Kant zu Intuition und Imagination}

An dieser Stelle muss die Stellung Immanuel Kants zur (genuinen) Intuition neu beurteilt werden. Im philosophiegeschichtlichen Durchgang wurde Kant noch die Rolle des Intuitionsskeptikers zugewiesen. Doch zugleich wird Kant aufgrund seines Imaginationsbegriffs überraschenderweise zum Wegbereiter des hier vertretenen Intuitionsverständnisses. Wie ist dies möglich?

Mentale Modelle sind psychologisch ausdifferenzierte Imaginationen in Kants Sinn: Hervorbringungen der produktiven Einbildungskraft, die zwischen Anschauungen und Begriffen vermitteln. Sie bündeln die empirischen Anschauungen, damit diese unter Begriffe subsumiert werden können

405 Zum psychologischen Essentialismus siehe Gelman (2003, insb. Kap. 11).

406 Vgl. Gelman (2003, S. 302). 
(Apprehension). ${ }^{407}$ Peirces Überlegungen zu Diagrammen und Johnson-Lairds Ansatz der Mentalen Modelle können als Konkretisierungen der Kantischen Konzeption der produktiven Einbildungskraft bzw. Imagination gelten. ${ }^{408}$ Die Verbindungsachse Kant-Peirce-Johnson-Laird wird außerdem durch die Unterscheidung zwischen Gemein- und Wesensbegriffen und die damit verbundene Rolle der mentalen Modelle/Imaginationen gestützt. Da die mentalen Modelle/Imaginationen das durch Rezeptivität Aufgenommene bündeln, ermöglichen sie es, durch von diesen mentalen Modellen ausgehendes Typ 1-Denken zu Wesensbegriffen zu kommen. Hierbei ist >Wesensbegriff< allerdings nicht mehr derart metaphysisch befrachtet wie bei Spinoza, sondern bezeichnet Intuitionen ${ }_{\mathrm{NP}}$, also Ableitungen aus mentalen Modellen. Diese sind die menschenmöglichen (quasi-)Wesensbegriffe.

Warum aber weist Kant die Möglichkeit genuiner Intuition zurück, wenn die genannten Elemente seiner eigenen Philosophie die Intuition gerade zu befördern scheinen? Dies liegt daran, dass Imagination realitäts-insensitiv ist: Keine Imagination muss der Realität entsprechen. Dazu passend bringt Kant im Rahmen seiner Ästhetik die Einbildungskraft (= Imagination) in Zusammenhang mit Dichtung und Phantasie. Zugleich folgt Kant noch dem klassisch-rationalistischen Intuitionsverständnis, wenn er den intuitiven Verstand mit dem göttlichen gleichsetzt und ihm die Erkenntnis des Noumenon (also des wahren Seienden) zuordnet. ${ }^{409}$ Dagegen wurde in dieser Arbeit der Infallibilitätsanspruch der Intuition, oder gar der etwas schwächere Anspruch, Intuition sei eine per se privilegierte Erkenntnisart, bewusst aufgegeben, ohne die spezifischen Stärken der Intuition zu verneinen. Dadurch können Kants Ausführungen zur Imagination als Stütze des Intuitionsbegriffs aufgenommen werden, ohne zugleich seine Eingrenzung der Intuition auf empirische Anschauung zu unterstützen.

Noch einzuordnen bleibt das hier entwickelte Intuitionsverständnis hinsichtlich Kants Differenzierungen zwischen intellektueller Intuition, empirischer Intuition, intuitivem Verstand und diskursivem Verstand. Kant ist der Auffassung, dass der Mensch weder über eine intellektuelle Anschauung noch über einen anschauenden Verstand verfügt. Die Möglichkeit intellektueller Anschauung verneint Kant mit dem Hinweis, Anschauung sei gänzlich rezeptiv und könne dadurch niemals selbsttätig einen Begriff

\footnotetext{
407 Vgl. KrV A 120.

408 Vgl. Klausen (2006, S. 94f.).

409 Vgl. KrV B 307.
} 
hervorbringen. ${ }^{410}$ Sinnlichkeit ist demnach für diese Spontaneität der Begriffsbildung stets auf den diskursiven Verstand angewiesen, weshalb der diskursive Verstand nur mit Gemeinbegriffen und nicht etwa mit Wesensbegriffen operiere. Die abduktive Methode, wie sie oben im Zusammenhang mit Peirce beschrieben wurde, existiert bei Kant somit nicht oder lässt sich zumindest nicht als sinnvoller Übergang von Anschauungen zu Wesensbegriffen verstehen.

Wie steht die vorliegende Studie zur Frage nach der Möglichkeit intellektueller Anschauung? Die Antwort scheint ernüchternd: Hier wurde keine Position dazu erarbeitet. Es kann aber gut begründet werden, warum dies nicht erfolgte: Die Frage nach der intellektuellen Anschauung ist keine Frage nach der Intuition, zumindest nicht im Sinn des hier erarbeiteten Verständnisses. Auch diese Frage lässt sich vertieft und mit Einbezug zeitgenössischer psychologischer Kenntnisse angehen, betrifft dann aber die Wahrnehmung im engeren Sinn: Es geht darum, inwiefern Wahrnehmungen bereits kognitiv durchdrungen sind. Ein an Peirce anknüpfendes Beispiel für Arbeiten in dieser Richtung sind Untersuchungen zur visuellen Abduktion. ${ }^{411}$ Ein älteres Beispiel hierfür sind Schopenhauers Argumente für die Intellektualität der Wahrnehmung. ${ }^{42}$ Mit der Konzeption einer von mentalen Modellen ausgehenden Intuition bewegt sich die hier erarbeitete Position auf einer anderen Ebene. Sie bleibt neutral hinsichtlich der Frage, wieviel Intellektualität wir bereits der Anschauung beimessen wollen. Prinzipiell kann weiterhin ein Kantianischer, d.h. rein rezeptiver Anschauungsbegriff vertreten werden, ohne mit den hier vertretenen Ausführungen zur Intuition in Konflikt zu geraten.

Anders sieht es mit Kants Eingrenzung des menschlichen Verstandes auf einen diskursiven Verstand aus: Hier ist das Thema der Untersuchung getroffen - und zwar im widersprechenden Sinn. Mit der Einordnung der Intuition als im Wesentlichen nichtbegrifflicher Vorgang, der jedoch begriffliche Ausgangspunkte und v.a. Resultate haben kann, wurde - gegen die Kantianische Position - Intellektualität jenseits des diskursiv-begrifflichen Bereichs postuliert. Das Anschauende des >anschauenden Verstands $<$ ist dabei metaphorisch zu verstehen. Es ist kein perzeptiver Vorgang, wenn mentale Modelle abgelesen werden. Intuition ist also nicht mit visueller Wahrnehmung gleichzusetzen - dagegen wurde u.a. im Zusammenhang mit der Kritik an Bealers und Chudnoffs Sicht der Intuition als Erscheinung argumentiert. Der

\footnotetext{
$410 \quad$ Vgl. KrV B 68.

411 Vgl. Magnani (2001, S. 106ff.).

412 Siehe Kap. 2.4.
} 
Vorgang der Intuition hat aber mit der Perzeption einiges gemeinsam: die Ganzheitlichkeit des Erfassens, die Reichhaltigkeit (das mentale Modell ist immer reichhaltiger als begriffliche Beschreibungen, die sich auf dieses mentale Modell beziehen) und die Äußerungen der Intuition auf der phänomenalen Ebene (Unmittelbarkeit und Gewissheit). In diesem übertragenen Sinn soll Kants Terminus >anschauender Verstand aufgefasst werden, damit er zum hier entwickelten Intuitionsverständnis passt.

Diese Passung kann nun explizit gemacht werden. Der Verstand kann deshalb anschauend sein, weil ihm durch Apprehension Imaginationen (= mentale Modelle) gegeben werden, die er ablesen kann. >Ablesen< heißt, dass er auf unmittelbarem, d.h. nicht diskursiv vermitteltem Weg erfassen kann, was in den mentalen Modellen enthalten ist. Insofern der Verstand in dieser Tätigkeit begriffen ist, geht er intuitiv vor. Eine Facette des Verstandes (die intuitive) beutet also quasi aus, was die andere (die Apprehension) durch die Konstruktion der mentalen Modelle hervorgebracht hat. Diese Doppelrolle des Verstandes wirft nochmals Licht auf die Frage nach der Aktivität/ Spontaneität bzw. Passivität/Rezeptivität der Wahrnehmung und Begriffsbildung. Der Verstand ist aktiv/spontan, aber nicht bei der Begriffsbildung, sondern bereits zuvor, nämlich bei der Apprehension. ${ }^{413}$ Dass Apprehension damit ebenso willkürlichen Faktoren unterworfen sein kann wie die diskursive Begriffsbildung, wird durch die Fallibilität dieser Art der Konstruktion mentaler Modelle erwiesen (siehe etwa die Diskussion zu stereotypischen bis rassistischen Weisen der Typisierung, bei denen der intellectus archetypus die Wahrheit weit verfehlen kann). Die Intuition behält auch in der hier vertretenen Auffassung die Merkmale der Rezeptivität und Realitätssensitivität, wobei diese in Bezug auf mentale Modelle verstanden werden müssen. Die Frage nach der Korrektheit der mentalen Modelle (in Bezug auf die Realität) bleibt zunächst ausgeklammert, da deren Korrektheit die Rezeptivität der Intuition nicht beeinflusst: In jedem Fall ist sie das Resultat einer Ableitung aus mentalen Modellen. Die Möglichkeit des intuitiven Verstandes ist somit die Möglichkeit eines Verstandes, der sich sowohl aktiv/spontan als auch passiv/rezeptiv verhält.

413 Hierzu passend Kant:

Es ist eine und dieselbe Spontaneität, welche dort unter dem Namen der Einbildungskraft, hier des Verstandes, Verbindung in das Mannigfaltige der Anschauung hineinbringt.

(KrV B 162 Anm.) 
Was ist Intuition? Können Intuitionen epistemische Gründe sein? Ziel der Untersuchung war, diese beiden Fragen zu beantworten. Die Kernresultate sollen im Folgenden zusammengefasst und mit einem Ausblick ergänzt werden.

In Bezug auf die erwähnten Fragen habe ich acht Kernthesen vertreten (siehe Kap. 1). Dabei hat sich folgendes Grundverständnis der Intuition herausgebildet: Intuition ist eine Form der Erkenntnis, die sich maßgeblich auf der nichtpropositionalen Ebene abspielt. Dieser nichtpropositionale Vorgang lässt sich genauer als Ableitung des Typ 1-Denkens beschreiben, die ausgehend von mentalen Modellen zu nichtpropositionalen Intuitionen (Intuitionen $\mathrm{NP}_{\mathrm{NP}}$ ) führt.

Mit einem solchen Intuitionsverständnis sind zwei Theorien der kognitiven Psychologie verbunden. Der Terminus >Typ 1< stammt aus der Dual-ProzessTheorie, die schnelles, unbewusstes und unmittelbares Denken dem langsamen, bewussten und schrittweisen Denken gegenüberstellt und somit auf Basis empirischer Evidenz die klassisch-philosophische Unterscheidung zwischen intuitivem und diskursivem Denken fortschreibt. Die Theorie der mentalen Modelle postuliert, dass Menschen Sachverhalte durch mentale Modelle repräsentieren. Diese mentalen Modelle werden insbesondere bei Schlussfolgerungen bedeutsam. Das Konzept der mentalen Modelle schließt u.a. an die Überlegungen Kants (produktive Imagination) und Peirces (Ikonizität) an. Die Reichhaltigkeit der mentalen Modelle führt dazu, dass das Typ 1-Denken diese bereits vorhandenen Repräsentationen »ablesen « und daraus Weiteres ableiten kann. Das so Abgeleitete können wir treffend als Intuitionen bezeichnen. Diese sind zunächst nichtpropositionale Gehalte (Intuitionen $\mathrm{NP}_{\mathrm{NP}}$.

Die Intuitionen $\mathrm{NP}_{\mathrm{N}}$ konstituieren Neigungen zu Überzeugungen. Dies ist so zu verstehen: Falls deren Gehalt durch Typ 2-Denken aufgenommen wird, bildet das Subjekt ceteris paribus eine der Intuition entsprechende Überzeugung (kurz: intuitive Überzeugung), welche die phänomenalen Merkmale der Unmittelbarkeit und Gewissheit aufweist. Die intuitive Überzeugung ist eine von zwei möglichen Arten der propositionalen Intuition. Die andere Art ergibt sich daraus, dass die Neigung zu einer Überzeugung, die durch die basale Intuition konstituiert wird, durch eine gegenläufige Überzeugung überstimmt wird, wie dies z.B. beim Mathematiker mit der Spielerfehlschluss-Intuition der Fall ist. Diese Art propositionaler Intuition wurde hier als >Intuition, dass $\mathrm{p}<$ bezeichnet, um sie von der intuitiven Überzeugung abzugrenzen. Vielfach 
gerät nur die Ebene der propositionalen Intuition ins Blickfeld der philosophischen Arbeiten zur Intuition. Daraus, dass wir Wendungen wie »er hatte die Intuition, dass $\mathrm{p}$ « meist gut nachvollziehen können, wird auf die generelle Propositionalität der Intuition geschlossen. Dies ist ein Kurzschluss, da so die Genese der Intuition, die für das Verständnis dieses mentalen Zustands charakteristisch (da für dessen phänomenalen Merkmale verantwortlich) ist, ausgeblendet wird.

Intuitionen können Gründe sein. Mit dieser Annahme, fokussiert auf den epistemischen Bereich, wurde der zweite Hauptteil der Arbeit eröffnet (Kap. 4). Hier wurde der Zusammenhang zwischen den basalen Intuitionen und den intuitiven Überzeugungen auf propositionaler Ebene untersucht. Es war dabei zu zeigen, wie die basalen Intuitionen, also nichtbegriffliche Gehalte, propositionale Überzeugungen rechtfertigen können. Dies führte zum allgemeinen Problem nichtbegrifflicher Rechtfertigung und damit zu einer Auseinandersetzung mit Vertretern einer Ansicht, wonach nur begriffliche Gehalte genuine Gründe für Subjekte sein können (v.a. McDowell). Gegen diese Ansicht wurde geltend gemacht, dass sinnvoll von basalen Intuitionen als Gründen gesprochen werden kann, ohne eine rein externalistisch-naturalistische Rechtfertigungstheorie zu vertreten oder dem »Mythos des Gegebenen« anheimzufallen. Dieser Weg ist gangbar, weil nichtbegriffliche Gehalte die Adäquatheitsbedingungen, die sinnvollerweise an begründende Gehalte gestellt werden, erfüllen können.

Damit Intuitionen Gründe sein können, müssen sie sowohl externalistischen als auch internalistischen Anforderungen gerecht werden. Aus der externalistischen Perspektive sind Intuitionen dann Gründe, wenn sie zuverlässig gebildet wurden. Für die Beurteilung dieser Zuverlässigkeit wurde ein auf der Adaptation der jeweiligen Intuition beruhender Vorschlag skizziert. Aus der notwendigerweise hinzuzufügenden internalistischen Perspektive wurden die Anforderungen an Gründe va. dadurch erfüllt, dass dem Subjekt via mentales Modell der relevante Sachverhalt bekannt ist, sowie dadurch, dass es die intuitive Überzeugung als intuitiv einordnen kann (mentale Demonstration).

Dieses Verständnis der Intuition und der intuitiven Rechtfertigung führte $\mathrm{zu}$ verschiedenen Betrachtungen des Verhältnisses zu anderen mentalen $\mathrm{Zu}-$ ständen und damit verbundener philosophischer Diskussionen. Intuitionen wurden dabei v.a. von Überzeugungen, Erscheinungen, Imaginationen und Formen der Irrationalität (Wunschdenken, Selbsttäuschung, Delusion) abgegrenzt, wobei auch Wechselwirkungen und Gemeinsamkeiten erkennbar wurden. Schließlich wurde auf Basis des postulierten Intuitionsverständnisses 
die aktuell geführte philosophiemethodische Debatte zum Status der Intuition kommentiert.

Abschließend möchte ich einige weitere Erkenntnisse und Ausblicke skizzieren, die sich aus dem Dargelegten ergeben, beginnend mit einer methodischen Bemerkung zur Herleitung des Intuitionsverständnisses. An einer Tagung wurde ich kürzlich von einem der experimentellen Philosophie zugeneigten Kollegen mit einer Idee konfrontiert, die ich hier paraphrasiere: »Wenn du im ersten Teil der Arbeit wissen willst, was Intuition ist und es für dich offenbar eine Rolle spielt, wie der Begriff heute verwendet wird, musst du wohl empirisch vorgehen: Die tatsächliche Begriffsverwendung der Menschen muss ermittelt werden. « Diese Bemerkung veranlasste mich dazu, nochmals über meine Methodik nachzudenken. Ich bin zu der Auffassung gelangt, dass der Kollege nicht völlig unrecht, aber nur bedingt recht hat. Der Art und Weise, wie der Begriff verwendet wird, kommt tatsächlich eine gewisse Autorität zu: Die dargestellten Beispiele, etwa dasjenige der Feuerwehrkommandantin, dienten u.a. dazu, das hier entwickelte Intuitionsverständnis an solche Alltagsverständnisse anschlussfähig zu machen. Damit ist die Hoffnung verbunden, dass viele Lesende bei diesen Beispielen den Eindruck haben, sie verstehen etwas Ähnliches unter Intuition - wodurch sie von den anschließenden Analysen der Fälle leichter überzeugt werden können. Bei einem solchen Vorgehen können empirische Untersuchungen Hilfestellungen bieten, um noch treffendere oder zusätzliche Aspekte abdeckende Beispiele zu finden, die gut an verbreitete Verständnisse anschließen. Doch niemals wäre die Begriffsarbeit durch eine empirische Erhebung ersetzbar, denn jene Arbeit geht entschieden weiter: Aufgrund der Kernverwendungen des Begriffs wird das fragliche Phänomen in den Blick genommen und vertieft betrachtet. Zunächst muss erwiesen werden, dass >Intuition « nicht ein beliebig zusammengesetztes begriffliches Konstrukt ist, sondern eine mentale Gegebenheit bezeichnet. ${ }^{414}$ Nach dieser Arbeit muss auf die Ebene der alltäglichen Begriffsverwendung zurückgekehrt und diese kritisch reflektiert werden. Hieraus kann sich die Erkenntnis ergeben, dass die Begriffsverwendung dem Phänomen nicht vollständig entspricht.

Ich möchte also eine methodische Pendelbewegung in der philosophischen Arbeit vorschlagen. Erstens: Wir beginnen bei den Begriffen

$414>$ Mentale Gegebenheit< bezieht sich hier auf die phänomenale Ebene. Es bleibt an dieser Stelle offen, wie Intuition realisiert ist. Multiple Realisierungen (evtl. durch ganz unterschiedliche Gehirnprozesse) sind eine Möglichkeit; die vorliegende Arbeit beschäftigt sich nicht mit dieser Ebene und lässt solches offen. 
(Alltagsverwendungen, philosophische und psychologische Verwendungen) und dringen durch Verdichtung zu den beschriebenen Phänomenen vor. Zweitens: Wir bestimmen diese Phänomene genauer, also notwendige und hinreichende Bedingungen, Bezüge und Abgrenzungen zu anderen Phänomenen, usw. Drittens: Wir kehren zu der Ebene der Begriffsverwendung zurück und modifizieren diese gegebenenfalls. Ich vermute, dass dieses Vorgehen auch für andere Begriffe und Phänomene gewinnbringend eingesetzt werden kann; insbesondere für solche, die sowohl in der philosophischen Tradition als auch in alltäglichen Kontexten prominent auftreten.

Nun zu zwei inhaltlichen Bemerkungen im Anschluss an die vertretenen Thesen: Erstens möchte ich kurz auf den möglichen Vorwurf des Psychologismus zu sprechen kommen, der gegen jene Thesen erhoben werden könnte. Ein Problem der Arbeit bestünde demnach darin, dass hier psychologisch erklärt wird, wie Intuition funktioniert und daraus erkenntnistheoretische Konsequenzen abgeleitet werden, ohne etwa den Unterschied zwischen Genese und Geltung zu beachten: Daraus, wie Intuition funktioniert, folgt der Kritik zufolge nichts über deren epistemischen Status.

In einem Antwortansatz auf diesen Vorwurf lässt sich dessen Voraussetzung hinterfragen: Es ist keineswegs offensichtlich, dass psychologische (oder allgemeiner: empirische) Tatsachen keinen Beitrag zur Beurteilung des epistemischen Status der Intuition leisten können. Insbesondere in Bezug auf die externalistische Rechtfertigungsfrage zeigte sich, dass für die Beurteilung der Zuverlässigkeit der Intuition Fakten bezüglich des Subjekts, dessen Umwelt und der Intuition als mentaler Zustand mindestens gewinnbringend, oftmals sogar notwendig einzubeziehen sind. Auch aufgrund der Theorie der mentalen Modelle konnten psychologische und philosophische Perspektiven sinnvoll miteinander verbunden werden. Psychologische Tatsachen können etwa aufzeigen, dass ein philosophisches Konzept eingesetzt werden kann, um in der Beantwortung einer Frage voranzukommen: Dies ist im Fall der mentalen Modelle das auf Russell zurückgehende Konzept der Bekanntschaft, mit dem erwiesen werden kann, dass Intuitionen die Anforderungen an Gründe erfüllen können. Erst die psychologische Stützung durch den Mentale-Modelle-Ansatz macht die Anwendung des Bekanntschaftskonzepts plausibel. Perspektiven der empirischen Wissenschaften und der Philosophie können in vielen Fragen der menschlichen Erkenntnis sinnvoll miteinander verbunden werden, um Fortschritte in deren Beantwortung zu erzielen.

Zur zweiten inhaltlichen Schlussbemerkung: Der hier ausgearbeitete Ansatz nichtbegrifflicher Begründung ließe sich vermutlich auf die intensiv geführten Diskussionen um nichtbegriffliche Gehalte der Wahrnehmung übertragen. So schließt diese Arbeit an Überlegungen an, welche die 
Komplementarität begrifflicher und nichtbegrifflicher Erkenntnis betonen oder die nichtbegriffliche Erkenntnis als fundamental erachten. ${ }^{415}$ Solche Ansätze sehen sich auch in den Bereichen wie Wahrnehmung oder Introspektion mit der Herausforderung konfrontiert, wie entsprechende Gehalte Gründe sein können: Wenn dafür eine Propositionalisierung erfolgen muss, wie ist diese zu denken? Wenn nicht, wie lässt sich die Begründungsrelation denken, ohne in Kohärentismus (nichtbegriffliche Gehalte nur als kausale Inputs in ein begriffliches System zugelassen), Naturalismus (Reduktion der Normativität der Gründe) oder eine Version des »Mythos des Gegebenen« zu verfallen? Der hier an der Intuition erprobte Ansatz geht insofern einen neuen Weg, als er die Begrifflichkeits-Anforderung an Gründe hinterfragt. Er distanziert sich damit von den genannten Optionen und schafft Raum für eine genuine Begründungsrelation mit nichtbegrifflichen Ausgangspunkten. Dessen Vertiefung und Ausweitung auf andere Bereiche der menschlichen Erkenntnis scheint mir möglich und sinnvoll.

Ich hoffe, mit diesem Buch auch einen Beitrag zur Überwindung der traditionellen wie auch aktuellen Kluft zwischen »Kopf und Bauch« (viele alternative Begriffe bezeichnen jene verbreitete Dichotomie) geleistet zu haben. Mit der Hochachtung der Intuition als Erkenntnisart sollte also keinesfalls einem Irrationalismus das Wort geredet werden, im Sinne von: »Hört mehr auf euer Bauchgefühl!« Aus der hier vertretenen Perspektive muss diese Dichotomie vielmehr äußerst kritisch betrachtet werden. Im Bewusstsein bestehender Tendenzen, Gründe auf den Bereich des Natürlichen zu reduzieren oder im Gegenteil scharf davon abzugrenzen, wollte ich einen anderen Weg beschreiten: Gründe dort entdecken, wo sie Philosophinnen und Philosophen oftmals nicht anzutreffen glauben. Solche Versuche werfen zweifellos viele neue Fragen auf; doch die Tatsache, dass am Ende einer philosophischen Untersuchung neue Fragen stehen, sollte keine unerwartete sein.

415 Vgl. Schildknecht (2002, S. 13; 2003); Gabriel (2015a). 